\title{
A score that verifies adherence to a gluten-free diet: a cross-sectional, multicentre validation in real clinical life
}

\author{
Federico Biagi ${ }^{1 *}$, Paola Ilaria Bianchi ${ }^{1}$, Alessandra Marchese ${ }^{1}$, Lucia Trotta $^{1}$, Claudia Vattiato ${ }^{1}$, \\ Davide Balduzzi $^{1}$, Giovanna Brusco ${ }^{2}$, Alida Andrealli ${ }^{3}$, Fabio Cisarò ${ }^{3}$, Marco Astegiano ${ }^{3}$, \\ Salvatore Pellegrino ${ }^{4}$, Giuseppe Magazzù ${ }^{4}$, Catherine Klersy ${ }^{5}$ and Gino Roberto Corazza ${ }^{1}$ \\ ${ }^{1}$ Coeliac Centre/First Department of Internal Medicine, Fondazione IRCCS Policlinico San Matteo, University of Pavia, \\ P.le Golgi, 19, 27100 Pavia, Italy \\ ${ }^{2}$ Internal Medicine Unit, Ospedale Civile di Voghera, Voghera, Italy \\ ${ }^{3}$ Department of Gastro-Hepatology, AOU San Giovanni Battista Molinette, University of Turin, Corso Bramante 88, \\ 10126 Turin, Italy \\ ${ }^{4}$ Regional Center for Celiac Disease, University of Messina, Messina, Italy \\ ${ }^{5}$ Service of Biometry and Clinical Epidemiology, Fondazione IRCCS Policlinico San Matteo, Pavia, Italy
}

(Submitted 27 July 2011 - Final revision received 7 December 2011 - Accepted 7 December 2011 - First published online 10 February 2012)

\begin{abstract}
A dietary interview performed by expert personnel is the best method to check whether patients with coeliac disease follow a strict glutenfree diet (GFD). We previously developed a score based on four fast and simple questions that can be administered even by non-expert personnel. The aim of the present study is to verify the reliability of our questionnaire in a new cohort of patients. The questionnaire has a five-level score. From March 2008 to January 2011, the questionnaire was administered to 141 coeliac patients on a GFD, who were undergoing re-evaluation. The score obtained was compared with persistence of both villous atrophy and endomysial antibodies (EMA). The rate of lower scores was higher among the patients with persistence of either villous atrophy (Fisher's exact, $P<0 \cdot 001$; test for trend, $P<0.001$ ) or positive EMA (Fisher's exact, $P=0.001$; test for trend, $P=0 \cdot 018$ ). Given that the coeliac patients have been well instructed on what a GFD means and on how to follow it, our questionnaire is a reliable and simple method to verify compliance to a GFD.
\end{abstract}

Key words: Coeliac disease: Gliadin: Endomysial antibodies: Villous atrophy

Coeliac disease (CD), a gluten-induced chronic enteropathy, is a very common condition characterised by increased mortality $^{(1,2)}$. Although increased mortality is due to a combination of malignancy, infection and $\mathrm{CVD}^{(2,3)}$, it was realised almost 30 years ago that mortality was mainly due to malignancies induced by poor adherence to a gluten-free diet (GFD) ${ }^{(4,5)}$; to date, it is not yet clear what is the best method to check whether patients are on a strict GFD ${ }^{(6)}$. The 'gold standard' is currently represented by a time-consuming dietary interview performed by expert personnel $^{(6)}$.

We recently developed a score based on just four very simple questions that could be administered in less than 1 min even by non-expert personnel ${ }^{(7)}$. Since the impossibility of knowing exactly what and, above all, how much gluten a person eats has already been pointed out ${ }^{(8)}$, our questionnaire is not based on the detection of how much gluten a coeliac patient consumes, but it is based on the analysis of the strategy performed by the patient to avoid gluten consumption. It is, therefore, a score that bypasses the problems linked not only to inadvertent gluten intake but also to cultural differences between patients and doctors and dietitians, which make it virtually impossible to compare GFD adherence in different groups of patients.

Our previous study had some limitations. First, the necessity that the patient has been appropriately instructed by an expert dietitian as to what a GFD really means and the fact, not always the case, that the patient is telling the truth. Second, it was based on a longitudinal retrospective study, which means that patients answered the questionnaire some time after the serological and histological tests had been carried out and were not blinded, as they knew the serological and histological results before the survey. This may have introduced an information bias as patients who were found to be positive either by serology or histology were likely to have

Abbreviations: CD, coeliac disease; EMA, endomysial antibodies; GFD, gluten-free diet.

*Corresponding author: Dr F. Biagi, fax +39 382 502618, email f.biagi@smatteo.pv.it 
been recalled about their compliance to the diet and were more likely to have realised that they had made errors. Finally, the study was performed in a single-referral centre. Although our previous study made it possible to show that there was a strong correlation between score results and persistence of villous atrophy and coeliac serology, it certainly cannot be considered to be representative of the use of the score in a 'real-life' clinical setting.

We organised the present study to verify the reliability of our compliance to a GFD score in a multicentre way. Since it is based on the histological and serological results obtained in each single centre, without centralising the assessments altogether, our final results are very close to everyday clinical practice.

\section{Methods}

\section{The questionnaire}

The questionnaire and the rationale on which it was based have been extensively described elsewhere (Fig. 1) ${ }^{(7)}$. Briefly, it consists of just a few questions that could be administered in a few minutes even by non-expert personnel. The numerical result makes it possible to monitor the strictness of GFD compliance over time and to compare it between different groups of patients, regardless of ethnic group. Although the questionnaire was drawn up in the form of an algorithm based on our clinical experience, the importance of the questions we asked has also been stressed by other authors ${ }^{(9-11)}$. The final score of the questionnaire is made up of five levels $(0-4)$, which, from a clinical point of view, can be grouped into three levels. Patients with a score of 0 or 1 do not follow a strict GFD, whereas patients with a score of 2 follow a GFD but with important errors that require correction. Patients with a score of 3 or 4 follow a strict GFD. It should be noted that the final question, which in some countries cannot be asked because local coeliac societies do not provide lists of packaged gluten-free food, could be omitted without affecting the final result of the score.

\section{Patients}

Between March 2008 and January 2011, four centres participating in the project administered the questionnaire to 141 coeliac patients (108 females, mean age 34 (SD 15) years) on a GFD (median 27 months, 25th-75th percentile 15-73, range 6-298), who were undergoing clinical, histological and serological re-evaluation. All these patients had been found to be affected by CD on the basis of villous atrophy and positive tissue transglutaminase or endomysial antibodies (EMA). They all received a proper instruction on a GFD from an expert dietitian. Patients were not enrolled if they were found to be affected by $\mathrm{CD}$ on the basis of minimal intestinal lesions or in spite of negative coeliac antibodies, or known to be suffering from refractory or complicated CD. Patients with IgA deficiency were also excluded from the study.

After signing written informed consent, patients underwent upper gastrointestinal endoscopy with multiple duodenal biopsies and EMA testing. The study was conducted according to the guidelines laid down in the Declaration of Helsinki and all procedures involving human subjects/patients were approved by the local review board. To avoid information bias, both at patient and doctor levels, we administered the questionnaire just before the clinical re-evaluation. The period to which the questionnaire is referred to is the same as that of the GFD of each patient. The validity of the questionnaire was assessed by measuring responsiveness to the clinical condition of the patients. First, the score was assessed against the persistence of positive EMA while on a GFD. The indirect

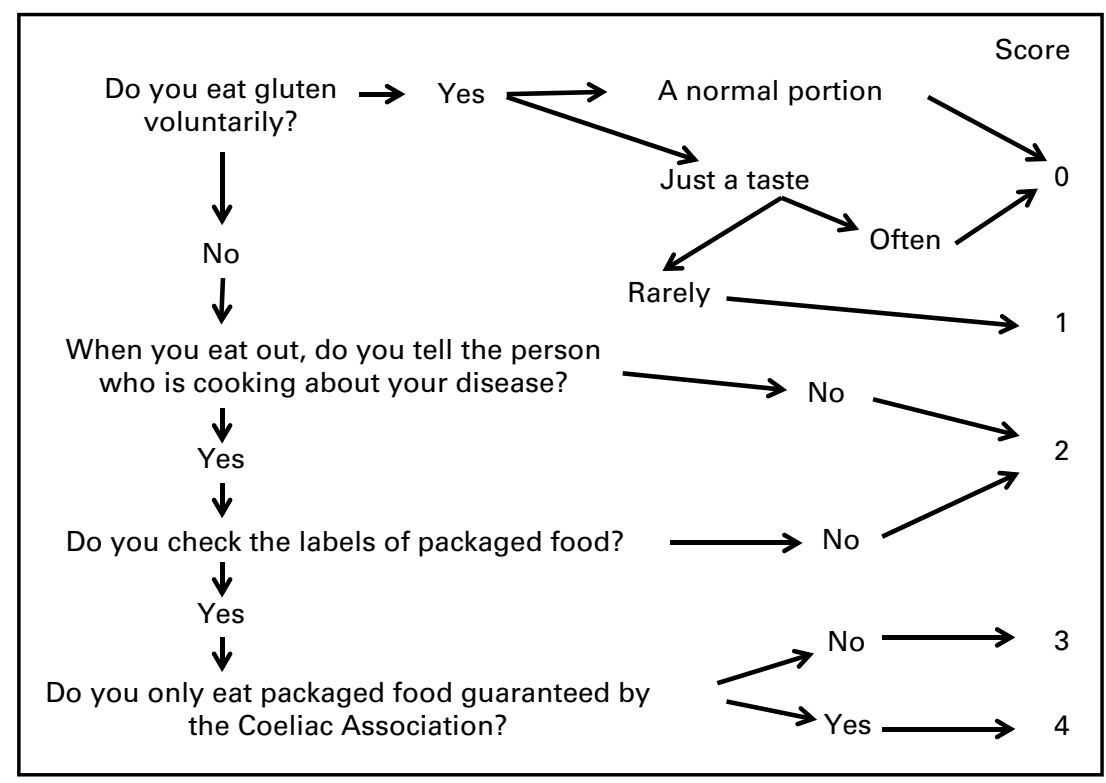

Fig. 1. Questionnaire and scoring system to assess compliance with a gluten-free diet in coeliac patients. 'Often': the patient consumes gluten so often that he/she cannot remember when and how many times that has happened. 'Rarely': the patient consumes gluten only occasionally. She/he can remember when and how many times that has happened. 
immunofluorescence assay was used to detect IgA EMA on monkey oesophagus sections ${ }^{(12)}$. Patients were classified as either EMA positive or none. The score was also assessed against persistence of villous atrophy at duodenal biopsy while on a GFD. According to O'Mahony et $a l .{ }^{(13)}$, we did not use complete intestinal healing as a proof of adherence to a GFD. A significant improvement in duodenal biopsy was considered to be satisfactory ${ }^{(13)}$. Therefore, using either Marsh or any other morphological classification of coeliac intestinal lesions to distinguish the biopsies would have been pointless. Persistent atrophy of the duodenal mucosa was thus used to classify the patients.

\section{Statistical analysis}

The association of the score with patient characteristics was assessed by means of Fisher's exact test and the test for trend. Exact logistic regression was used to compute OR and 95\% CI. Positive and negative predictive values (and 95\% CI) were computed as a measure of suspicion of histological abnormalities or EMA positivity for a series of possible scenarios (two cut-offs for the score and four possible prevalences of the anomaly). Stata 11 (Stata Corporation) was used for computation. All tests were two-sided. A $P$ value $<0.05$ was considered to be statistically significant.

\section{Results}

Table 1 shows the association between the score and the persistence of villous atrophy and EMA positivity. OR and 95\% CI were consistent with a very strong association between the score and the outcome of villous atrophy or EMA persistence. In addition, lower scores were associated with a higher rate of adverse findings. Despite being on a GFD, thirty-eight patients were still EMA positive at the time of duodenal biopsy. Patients with the persistence of EMA positivity more frequently showed the lowest scores. Of the 141 patients, twenty presented persistence of villous atrophy at the time of duodenal biopsy despite being on a GFD. Patients with the persistence of villous atrophy more frequently showed the lowest scores.

Table 2 shows the positive and negative predictive values for not only the original data but also for the four different prevalences of the anomaly and for the two possible cut-offs of the score.

The histological results were coherent with the score in the vast majority of patients, with scores $0-1$ or scores $3-4$ (126 out of $141,89 \cdot 3 \%$ ). Discrepancies were found in only fifteen patients scoring $0-1$ or $3-4(10 \cdot 7 \%)$. Of these fifteen patients, seven were found to have a restored duodenal mucosa despite a score of 0 or 1 . It is possible that voluntary ingestion of gluten had occurred much earlier than the clinical reevaluation, and so the mucosa had had time to recover (unfortunately, when we planned the present study, we did not think of recording when the gluten ingestion had occurred). The other eight patients were found to have a flat duodenal mucosa in spite of a score of 4 . Since three of them also were found to be positive to EMA, it is possible that they did not report the entire truth during the interview. On the other hand, the last five patients with villous atrophy but negative EMA are currently under investigation to verify whether they are affected by either a slow-responder or a refractory form of $\mathrm{CD}$. A patient scoring 0 died because of complicated CD. Finally, although a score of 2 indicates a poor compliance to a GFD that needs to be improved, only one patient showed persistence of villous atrophy. However, only seven patients scored 2 , and so it is quite difficult to draw any conclusions on this score level.

\section{Discussion}

In order to validate compliance to a GFD score, similarly to our retrospective study ${ }^{(7)}$, we assessed its responsiveness against both histological and serological responses to a GFD. Histology is certainly the most valid method for evaluating compliance with a GFD ${ }^{(6,14)}$, and, although serology is not sensitive enough to detect occasional intake of gluten ${ }^{(15)}$, it

Table 1. Association of the grouped score with patient characteristics (Odds ratios and $95 \%$ confidence intervals)

\begin{tabular}{|c|c|c|c|c|c|c|c|c|}
\hline \multirow[b]{2}{*}{ GFD score } & \multirow[b]{2}{*}{$n$} & \multicolumn{2}{|c|}{ EMA positive on a GFD } & \multirow[b]{2}{*}{$P^{*}$} & \multirow[b]{2}{*}{$P \dagger$} & \multirow[b]{2}{*}{ OR } & \multirow[b]{2}{*}{$95 \% \mathrm{Cl}$} & \multirow[b]{2}{*}{$P \S$} \\
\hline & & $n$ & $\%$ & & & & & \\
\hline $0-1$ & 18 & 11 & 61 & & & - & & - \\
\hline 2 & 7 & 0 & 0 & 0.001 & 0.018 & 0.09 & $0.00,0.74$ & 0.023 \\
\hline \multirow[t]{2}{*}{$3-4$} & 116 & 27 & 23 & & & 0.23 & $0.06,0.69$ & 0.007 \\
\hline & & \multicolumn{2}{|c|}{ Villous atrophy on a GFD } & & & & & \\
\hline GFD score & $n$ & $n$ & $\%$ & $P^{*}$ & $P \dagger$ & OR & $95 \% \mathrm{Cl}$ & $P \|$ \\
\hline $0-1$ & 18 & 11 & 61 & & & - & & - \\
\hline 2 & 7 & 1 & 14 & $<0.001$ & $<0.001$ & 0.04 & $0.00,0.75$ & 0.024 \\
\hline $3-4$ & 116 & 8 & 7 & & & 0.02 & $0.00,0.10$ & $<0.001$ \\
\hline
\end{tabular}

GFD, gluten-free diet; EMA, endomysial antibodies.

* Fisher's exact test.

$\dagger$ Trend test.

$\ddagger$ Exact logistic regression, stratified by centre.

$\S$ Model $P=0.002$.

\| Model $P<0.001$. 
is certainly one of the most commonly used methods ${ }^{(6)}$. On the other hand, we did not assess it against clinical response to a GFD. Apart from the fact that many patients are nowadays asymptomatic and are found to be affected by CD because of serological screening on relatives or associated autoimmune conditions, we and others ${ }^{(14,16)}$ have shown that clinical response to a GFD is not invariably associated with mucosal recovery, and we have recently shown that clinical response to a GFD can occur even in non-coeliac patients ${ }^{(17)}$. One could argue that our system simply reflects a subjective evaluation. In our initial retrospective evaluation, we showed that there was a considerable discrepancy between score and subjective evaluation ${ }^{(7)}$. Finally, we were unable to make a comparison with a classic dietary interview, as this would have included an information bias in the study: patients will know the result of the first interview and this will inevitably influence his/her answers to the second one.

In the present study, we showed that our system does indeed verify adherence to a GFD in coeliac patients. We were very impressed to see that the present results are even better than those we obtained in our preliminary, retrospective and single-centre study ${ }^{(7)}$. Moreover, it should be noted that the results we showed here are the sum of all the results obtained independently by the four centres. Moreover, patient education about CD, level of education and socio-economic status were not uniform and were not standardised. So, the present results can certainly be considered to be representative of what happens in real clinical life. Having found good negative predictive values further supports that our system does really identify patients without the persistence of EMA positivity and villous atrophy.

All this evidence clearly shows that our score is a reliable tool to check GFD adherence in coeliac patients. It is extremely rapid and simple and can also be administered even by personnel without specific experience in CD. However, this does not reduce the importance of expert dietitians who have the initial role of educating new coeliac patients regarding a GFD ${ }^{(18)}$. We underline that our score can be applied only to patients who have been well instructed on what a GFD means and on how to follow it.

The numerical result would thus make it possible to monitor the patient's compliance with the GFD over time and to make comparisons between different groups of patients. This possibility of comparing different groups of patients is made even easier by the fact that our score is not based on an assessment of what the patient eats, which would make it very difficult to compare patients from different countries or with different cultural backgrounds, but on the strategies that the patient uses to avoid eating gluten, regardless of the actual food eaten.

Finally, in the initial retrospective validation, we assessed our score against the survival of patients ${ }^{(7)}$. In the present study, this was not possible because, fortunately, only one patient died. The patient was a 63-year-old lady who developed intestinal lymphoma shortly after the diagnosis of CD. She never followed a strict GFD and scored 0 at our questionnaire. 
In conclusion, our score is an extremely rapid, simple and reliable tool to check GFD compliance in CD patients. It can also be administered by personnel without specific experience in $\mathrm{CD}$.

\section{Acknowledgements}

We are grateful to Susan West for reading and correcting the manuscript. This study received no funding. F. B. designed the study, interpreted the results and wrote the manuscript. P. I. B., A. M., L. T., C. V. and D. B. administered the questionnaire to the patients in Pavia and acquired the clinical, serological and histological data. G. B. administered the questionnaire to the patients in Voghera and acquired the clinical, serological and histological data. A. A., F. C. and M. A. administered the questionnaire to the patients in Turin and acquired the clinical, serological and histological data. S. P. and $G$. M. administered the questionnaire to the patients in Messina and acquired the clinical, serological and histological data. C. K. performed the statistical analysis. G. R. C. participated in the design of the study and in the critical revision of the manuscript. The authors declare that they have no conflicts of interest.

\section{References}

1. Biagi F, Klersy C, Balduzzi D, et al. (2010) Are we not over-estimating the prevalence of coeliac disease in the general population? Ann Med 42, 557-561.

2. Biagi F \& Corazza GR (2010) Mortality in celiac disease. Nat Rev Gastroenterol Hepatol 7, 158-162.

3. Grainge MJ, West J, Card TR, et al. (2011) Causes of death in people with celiac disease spanning the pre- and postserology era: a population-based cohort study from Derby, UK. Am J Gastroenterol 106, 933-939.

4. Leonard JN, Tucker WF, Fry JS, et al. (1983) Increased incidence of malignancy in dermatitis herpetiformis. BMJ $\mathbf{2 8 6}$, $16-18$.

5. Holmes GK, Prior P, Lane MR, et al. (1989) Malignancy in coeliac disease - effect of a gluten free diet. Gut 30, 333-338.
6. Pietzak MM (2005) Follow-up of patients with celiac disease: achieving compliance with treatment. Gastroenterology $\mathbf{1 2 8}$, S135-S141.

7. Biagi F, Andrealli A, Bianchi PI, et al. (2009) A gluten-free diet score to evaluate dietary compliance in patients with coeliac disease. Br J Nutr 102, 882-887.

8. Hopman EG, Kiefte-de Jong JC, le Cessie S, et al. (2007) Food questionnaire for assessment of infant gluten consumption. Clin Nutr 26, 264-271.

9. Leffler DA, Dennis M, Edwards George JB, et al. (2009) A simple validated gluten-free diet adherence survey for adults with celiac disease. Clin Gastroenterol Hepatol 7 , $530-536$.

10. See J \& Murray JA (2006) Gluten-free diet: the medical and nutrition management of celiac disease. Nutr Clin Pract 21, $1-15$.

11. Leffler DA, Edwards-George J, Dennis M, et al. (2008) Factors that influence adherence to a gluten-free diet in adults with celiac disease. Dig Dis Sci 53, 1573-1581.

12. Biagi F, Pezzimenti D, Campanella J, et al. (2001) Endomysial and tissue transglutaminase antibodies in coeliac sera. A comparison not influenced by previous serological testing. Scand J Gastroenterol 36, 955-958.

13. O'Mahony S, Howdle PD \& Losowsky MS (1996) Review Article: management of patients with non-responsive coeliac disease. Aliment Pharmacol Ther 10, 671-680.

14. Rubio-Tapia A, Rahim MW, See JA, et al. (2010) Mucosal recovery and mortality in adults with celiac disease after treatment with a gluten-free diet. Am J Gastroenterol. 105, $1412-1420$

15. Troncone R, Mayer M, Spagnuolo F, et al. (1995) Endomysial antibodies as unreliable marker for slight dietary transgressions in adolescents with coeliac disease. J Pediatr Gastroenterol Nutr 21, 69-72.

16. Biagi F, Campanella J, Martucci S, et al. (2004) A milligram of gluten a day keeps the mucosal recovery away. Nutr Rev $\mathbf{6 2}$, 360-363.

17. Campanella J, Biagi F, Bianchi PI, et al. (2008) Clinical response to gluten withdrawal is not an indicator of coeliac disease. Scand J Gastroenterol 43, 1311-1314.

18. Case S (2005) The gluten-free diet: how to provide effective education and resources. Gastroenterology $\mathbf{1 2 8}$, S128-S134. 